\title{
Perfil nutricional de colaboradores de uma instituição oncológica
}

\author{
Nutritional profile of employees of an oncology institution
}

\section{DOI: $10.37111 /$ braspenj.2020354014}

Melissa Sardenberg Rotatori'

Thais Manfrinato Miola ${ }^{2}$

Fernanda Ramos de Oliveira Pires

\section{Unitermos:}

Sobrepeso. Obesidade. Doença crônica. Saúde do trabalhador. Avaliação nutricional.

\section{Keywords:}

Overweight. Obesity. Chronic disease. Occupational health. Nutrition Assessment.

\section{Endereço para correspondência:}

Melissa Sardenberg Rotatori

A. C. Camargo Cancer Center - Centro Integral de Diagnóstico, Tratamento, Ensino e Pesquisa Rua Professor Antonio Prudente, 211 - Liberdade São Paulo, SP, Brasil - CEP 01509-900

E-mail: melissa_rotatori@hotmail.com

\section{Submissão:}

15 de dezembro de 2019

\section{Aceito para publicação}

7 de outubro de 2020

\begin{abstract}
RESUMO
Introdução: 0 excesso de peso e a obesidade são crescentes no mundo todo e estão relacionados ao desenvolvimento de doenças crônicas que podem trazer prejuízos à qualidade de vida pessoal e profissional. $O$ objetivo deste estudo foi traçar o perfil nutricional e hábitos de vida de colaboradores de uma instituição oncológica. Método: Estudo prospectivo, de coorte e observacional com 1.776 colaboradores do A. C. Camargo Cancer Center, em São Paulo (SP). Os dados foram coletados de setembro de 2017 a fevereiro de 2018, por meio de questionário e aferição de peso, altura, circunferência da cintura e índice de massa corpórea (IMC). Resultados: Quarenta e dois e meio por cento dos homens e $39,1 \%$ das mulheres estavam em sobrepeso de acordo com o IMC. O sexo feminino teve maior prevalência de classificação de risco muito elevado para circunferência da cintura $(45,1 \%)$. Os trabalhadores do turno noturno apresentaram maior prevalência de obesidade $(34,8 \%)$ e de risco muito elevado para doenças cardiovasculares $(47,1 \%)$ em relação aos outros turnos. Situação similar foi observada nos colaboradores que trabalham 11 anos ou mais na instituição, onde $27,9 \%$ estão obesos e $44,5 \%$ com risco muito elevado para doenças cardiovasculares, comparados aos que trabalham há menos tempo. Apenas $35,5 \%$ da amostra realizavam atividade física. Conclusão: A alta prevalência de sobrepeso e obesidade, bem como risco para doenças cardiovasculares encontrados na população estudada demonstra a necessidade e importância da adoção de estratégias para promoção hábitos alimentares mais saudáveis e prática de atividade física.
\end{abstract}

\section{ABSTRACT}

Introduction: Overweight and obesity are increasing in the world and are related to the development of chronic diseases that can impair personal and professional quality of life. The aim of this study was to track the nutritional profile and lifestyle of employees of an oncology institution. Methods: Prospective, cohort and observational study with 1,776 employees at the A. C. Camargo Cancer Center, in São Paulo (SP). Data were collected from September 2017 to February 2018, through a questionnaire and deferral of weight, height, waist circumference and body mass index (BMI). Results: Forty-two and a half percent of men and $39.1 \%$ of women were in excess according to the BMI. The female gender had a higher prevalence of very high risk classification for waist circumference (45.1\%). Night shift workers had a higher prevalence of obesity $(34.8 \%)$ and a very high risk for cardiovascular disease $(47.1 \%)$ compared to other shifts. Similar situation was observed in employees who work 11 years or more at the institution, where $27.9 \%$ are obese and $44.5 \%$ are at very high risk for cardiovascular diseases, compared to those who use less time. Only $35.5 \%$ of the sample performed physical activity. Conclusion: A high prevalence of obesity and obesity, and risk for cardiovascular diseases, found in the studied population, shows the need and importance of adopting strategies to promote healthier eating habits and physical activity practices.

1. Mestranda em Ciências na área de Oncologia pela FAP, Pós-graduação em Nutrição Clínica Funcional pela VP centro de nutrição funcional; Pós graduação em Nutrição Clínica pelo Ganep; Nutricionista clíniça no A. C. Camargo Cancer Center, São Paulo, SP, Brasil.

2. Doutoranda em Ciências na Área de Oncologia pela FAP; Mestrado em Ciências na Área de Oncologia pela FAP; Pós-graduação em Nutrição Clínica pela Faculdade CBES; Supervisora de Nutrição Clínica do A. C. Camargo Cancer Center, São Paulo, SP, Brasil.

3. MBA em Gestão da Saúde e Administração Hospitalar; Pós-graduação em Nutrição Clínica pelo Ganep; Graduação em nutrição pelo Centro Universitário São Camilo; São Paulo, SP, Brasil. 


\section{INTRODUÇÃO}

A transição econômica, seguida da industrialização, urbanização e globalização no século passado, veio associada a mudanças na dieta e no estilo de vida das pessoas, com profundas consequências sobre a saúde e o estado nutricional da população'.

Em 2016, havia 1,9 bilhões de adultos acima do peso, sendo que 650 milhões eram obesos². A Organização Mundial da Saúde (OMS), portanto, definiu a obesidade como a epidemia do século 21 , que tem alto impacto na morbimortalidade, saúde e qualidade de vida ${ }^{3}$.

A OMS também afirma que as doenças crônicas não transmissíveis são as principais causas de morte e incapacidade em todo o mundo 4 . No Brasil, o sobrepeso e a obesidade foram atribuídos a aproximadamente 168 mil mortes por ano relacionados às doenças crônicas ${ }^{5}$.

Em países desenvolvidos, o aumento do sobrepeso e da obesidade foi estabilizado na última década, enquanto que, em países em desenvolvimento, duas em cada três pessoas estão obesas, e sugere-se que este número tende a aumentar ${ }^{5}$. Além disso, em países desenvolvidos, os homens têm taxas mais elevadas de sobrepeso e obesidade, enquanto nos países em desenvolvimento, as mulheres apresentam taxas mais altas 6 .

No Brasil, houve um aumento contínuo de excesso de peso e obesidade na população com mais de 20 anos de idade (POF 2008-2009) ${ }^{7}$. Na pesquisa Vigitel, em 2018, foi encontrada a frequência de excesso de peso de $55,7 \%$, sendo maior entre homens $(57,8 \%)$ do que entre mulheres $(53,9 \%)^{8}$.

A causa fundamental da obesidade e do excesso de peso é um desequilíbrio energético entre as calorias consumidas e as calorias gastas. Mundialmente tem ocorrido um aumento da ingestão de grãos refinados, carne vermelha, gorduras não saudáveis e bebidas açucaradas, concomitante a uma baixa ingesta de grãos integrais, legumes, frutas e oleaginosas ${ }^{8,9}$.

O aumento da inatividade física também é uma causa da obesidade e ocorre devido à natureza cada vez mais sedentária no trabalho, transporte, tempo de lazer e nos hábitos rotineiros. Assistir à televisão, por exemplo, é um forte fator de risco, por ser uma atividade sedentária e também porque a exposição à publicidade de alimentos e bebidas pode influenciar na qualidade alimentar da população ${ }^{10}$

Além do desequilíbrio energético e da inatividade física, a obesidade também tem influência de fatores genéticos, ambientais, culturais e socioeconômicos. Fatores estes que podem criar um ambiente obesogênico, onde ocorrerá um aumento do consumo calórico e redução da atividade física, levando à obesidade?

O Programa de Alimentação do Trabalhador (PAT) é uma política pública com objetivo de melhoria do estado nutricional dos trabalhadores, partindo do pressuposto que indivíduos bem alimentados terão maior incentivo ao trabalho, gerando um impacto positivo na produtividade das empresas. O PAT foi estabelecido em 1976, quando os trabalhadores eram um grupo populacional vulnerável a doenças por deficiência. Sendo assim, há grande necessidade de revisão do PAT, para redefinir seus objetivos de acordo com a mudança do estado nutricional da população-alvo do programa, a fim de ajudar os trabalhadores a manter um peso corporal dentro de limites saudáveis ${ }^{11}$.

Como uma melhor qualidade de vida do trabalhador representa maior produtividade e maiores oportunidades de desenvolvimento, o intuito, portanto, é desenvolver a adoção de hábitos de vida saudáveis, ou seja, ser fisicamente ativo, ter uma alimentação saudável, limitar o consumo de álcool e cessar o tabagismo e ter bons hábitos de sono, o que também pode prevenir, pelo menos, $70 \%$ das principais doenças crônicas ${ }^{12}$.

O objetivo deste estudo foi traçar o perfil nutricional e hábitos de vida de colaboradores de uma instituição oncológica.

\section{MÉTODO}

O estudo realizado foi do tipo prospectivo, de coorte e observacional. A amostra foi calculada por conveniência e foi composta por 1.776 participantes do A. C. Camargo Cancer Center. A coleta de dados foi realizada no período de setembro de 2017 a fevereiro de 2018. O estudo foi previamente aprovado pelo Comitê de Ética do A. C. Camargo Cancer Center, sob o n 2416/17. Os critérios de inclusão foram: colaboradores do A. C. Camargo Cancer Center que concordaram em participar da pesquisa assinando o Termo de Consentimento Livre e Esclarecido; idade acima de 19 anos, ambos os sexos. Participantes que apresentavam impossibilidade de aferição de peso e/ou altura, assim como gestantes, foram excluídos da pesquisa.

Para a avaliação do estado nutricional dos participantes foi utilizado dados do índice de massa corpórea (IMC) e circunferência da cintura. $O$ peso e altura foram aferidos em balança com estadiômetro acoplado Filizola ${ }^{\circledR}$, conforme protocolo descrito por Dias et al. ${ }^{13}$. Os valores de referência que foram utilizados para o IMC para participantes com até 60 anos de idade foram da $\mathrm{OMS}^{14}$ e para participantes com idade superior a 60 anos, foram utilizados os valores de referência de OPAS (Organização Panamericana de Saúde) ${ }^{15}$. Para a aferição da circunferência da cintura, foi utilizada fita métrica não elástica e realizado conforme recomendação de Dias et al. ${ }^{13}$ e os valores de referência utilizados foram os propostos pela $\mathrm{OMS}^{14}$.

Os participantes também responderam a um questionário sobre seus dados sociodemográficos, doenças associadas, 
especificações do local de trabalho e nível de atividade física. O nível de atividade física foi classificado conforme critérios recomendados pela OMS ${ }^{16}$.

\section{Análise Estatística}

Para o processamento dos dados foi utilizado o software Statistical Package for Social Science (SPSS), versão 23. Foram utilizados os parâmetros da estatística descritiva, adotandose as medidas usuais de tendência central (média, mediana e moda) e cálculos de frequências simples e relativas. Para correlação entre as variáveis, foram utilizados os testes Qui-Quadrado e Teste Exato de Fischer para as variáveis categóricas. Testes T de Student e Mann-Whitney foram utilizados na análise de variáveis contínuas com distribuição normal e sem distribuição normal, respectivamente. $\bigcirc$ nível de significância adotado foi de $5 \%$.

\section{RESULTADOS}

O estudo foi composto por 1776 indivíduos, sendo $567(31,9 \%)$ do sexo masculino e $1209(68,1 \%)$ do sexo feminino, com idade mediana de 33 anos. A etnia branca foi predominante $(49,5 \%)$, assim como a área de trabalho de operações $(65,3 \%)$. A renda salarial mais prevalente foi de 1 a 3 salários mínimos $(49,7 \%)$. O sobrepeso foi mais prevalente $(40,2 \%)$, seguido da eutrofia $(34,8 \%)$, de acordo com o IMC. As características da amostra se encontram na Tabela 1. Além disso, os resultados da correlação entre classificação de IMC e classificação da circunferência da cintura são apresentados na Figura 1.

$\mathrm{Na}$ correlação da classificação da circunferência da cintura com o sexo, observamos diferença significativa $(p<0,0001)$, onde o sexo masculino teve maior prevalência de classificação adequada $(51,5 \%)$ e o sexo feminino teve maior prevalência de classificação de risco muito elevado $(45,1 \%)$. Porém quando a correlação foi feita com a classificação do IMC, não foi observada diferença significativa entre os sexos $(p=0,379)$ e tanto os homens quanto as mulheres tiveram maior prevalência de sobrepeso $(42,5 \%$ e $39,1 \%$, respectivamente).

A análise estatística demonstrou diferença significativa na comparação do tempo que trabalha na empresa e turno com a classificação da circunferência da cintura e do IMC. Os colaboradores do período noturno apresentaram maior prevalência de sobrepeso e obesidade (73,5\%), assim como risco elevado e muito elevado para doenças cardiovasculares $(72,9 \%)$ $(p=0,005$ e $p<0,0001$, respectivamente). Apenas 7,3\% da amostra relataram trabalhar 2 ou mais turnos. De forma similar, os colaboradores com 11 anos ou mais de empresa, onde o sobrepeso e obesidade $(71,5 \%)$ e risco elevado e muito elevado na circunferência da cintura $(76,8 \%)$ foram mais prevalentes ( $p<0,0001$ e $p<0,0001$, respectivamente).

\begin{tabular}{|c|c|c|}
\hline Variável & Categoria & $\mathrm{N}(\%)$ \\
\hline \multicolumn{3}{|l|}{ Sexo } \\
\hline & Masculino & $567(31,9)$ \\
\hline & Feminino & $1209(68,1)$ \\
\hline \multicolumn{3}{|c|}{ Idade (anos) } \\
\hline & Min-Max & $18-93$ \\
\hline & Mediana & 33 \\
\hline \multicolumn{3}{|l|}{ Etnia } \\
\hline & Branca & $880(49,5)$ \\
\hline & Parda & $578(32,5)$ \\
\hline & Negra & $282(15,9)$ \\
\hline & Indígena & $14(0,8)$ \\
\hline & Outra & $22(1,2)$ \\
\hline \multicolumn{3}{|l|}{ Peso } \\
\hline & Min-Max & $41,2-139$ \\
\hline & Mediana & 72,2 \\
\hline \multicolumn{3}{|l|}{ IMC } \\
\hline & Min-Max & $17,1-47,9$ \\
\hline & Mediana & 26,4 \\
\hline \multicolumn{3}{|c|}{ Classificação do IMC } \\
\hline & Desnutrição & $19(1,1)$ \\
\hline & Eutrofia & $618(34,8)$ \\
\hline & Sobrepeso & $714(40,2)$ \\
\hline & Obesidade & $425(23,9)$ \\
\hline \multicolumn{3}{|c|}{ Circunferência da Cintura } \\
\hline & Min-Max & $55-140$ \\
\hline & Mediana & 88 \\
\hline \multicolumn{3}{|c|}{$\begin{array}{l}\text { Classificação da Circunfe- } \\
\text { rência da Cintura }\end{array}$} \\
\hline & Adequada & $671(37,8)$ \\
\hline & Risco Elevado & $445(25,1)$ \\
\hline & Risco Muito Elevado & $660(37,2)$ \\
\hline \multicolumn{3}{|c|}{ Tempo de Trabalho em Anos } \\
\hline & Até 5 anos & $1133(63,8)$ \\
\hline & De 6 a 10 anos & $414(23,3)$ \\
\hline & Acima de 11 anos & $229(12,9)$ \\
\hline \multicolumn{3}{|l|}{ Cargo } \\
\hline & Diretoria Médica & $92(5,2)$ \\
\hline & Ensino e Pesquisa & $10(0,6)$ \\
\hline & Executiva Corporativa & $52(2,9)$ \\
\hline & Executiva de Negócios & $26(1,5)$ \\
\hline & Finanças & $93(5,2)$ \\
\hline & Foco do Paciente & $286(16,1)$ \\
\hline & Geral & $2(0,1)$ \\
\hline & Operações & $1159(65,3)$ \\
\hline & $\begin{array}{l}\text { Relações Institucionais } \\
\text { e Sustentabilidade }\end{array}$ & $10(0,6)$ \\
\hline & Recursos Humanos & $31(1,7)$ \\
\hline & Supply Chain & $15(0,8)$ \\
\hline
\end{tabular}




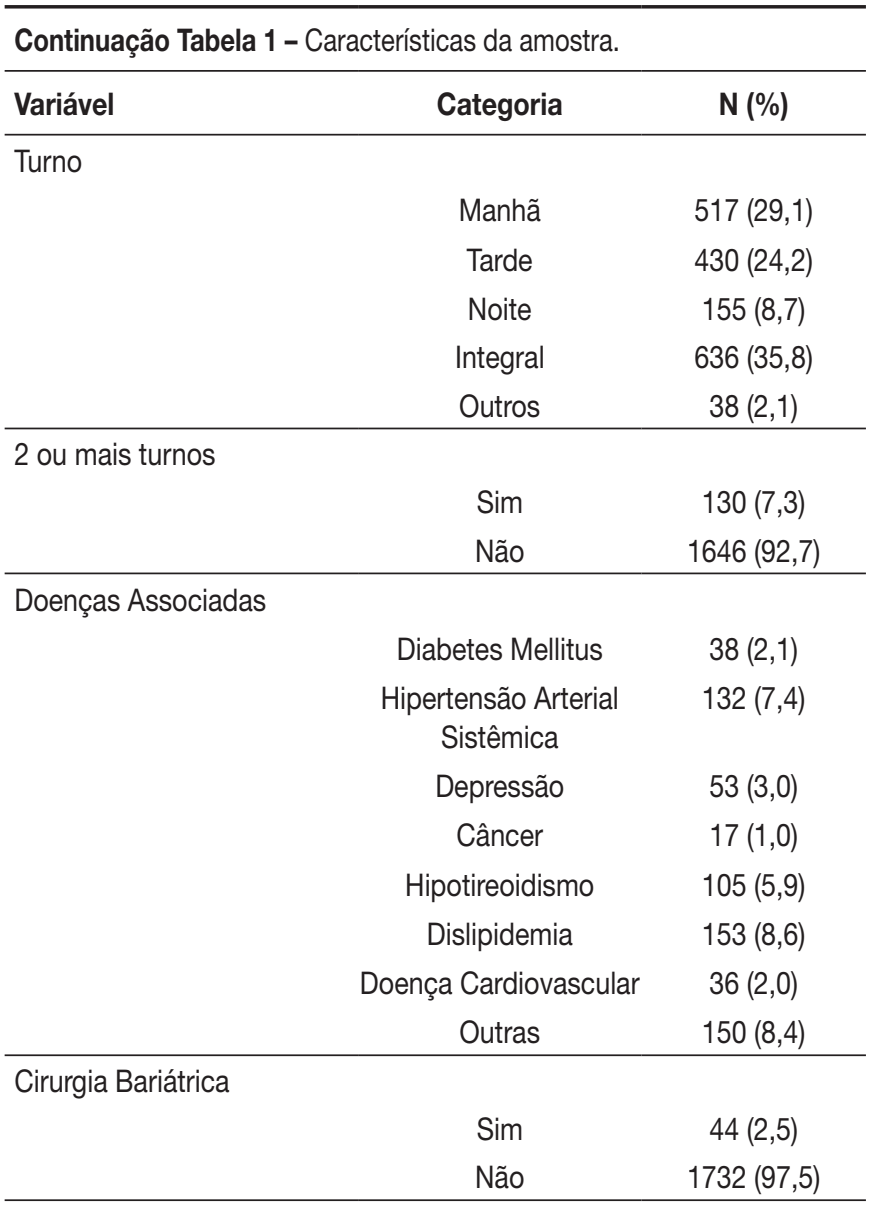

\begin{tabular}{|c|c|c|}
\hline \multicolumn{3}{|c|}{ Continuação Tabela 1 - Características da amostra. } \\
\hline Variável & Categoria & $\mathbf{N}(\%)$ \\
\hline \multicolumn{3}{|c|}{ Atividade Física } \\
\hline & Até 2 h29 & $227(35,9)$ \\
\hline & De 2h30 a 4 h59 & $195(30,9)$ \\
\hline & Mais de $5 \mathrm{~h}$ & $209(33,1)$ \\
\hline \multicolumn{3}{|l|}{ Hábitos } \\
\hline & Tabagismo & $128(7,2)$ \\
\hline & Etilismo & $574(32,3)$ \\
\hline \multicolumn{3}{|c|}{ Estado Civil } \\
\hline & Solteiro & $862(48,5)$ \\
\hline & Casado & $781(44,0)$ \\
\hline & Divorciado & $120(6,8)$ \\
\hline & Viúvo & $13(0,7)$ \\
\hline \multicolumn{3}{|c|}{ Grau de Instrução } \\
\hline & Sabe ler/escrever & $3(0,2)$ \\
\hline & Primeiro grau & $33(1,9)$ \\
\hline & Segundo grau & $717(40,4)$ \\
\hline & Superior & $1023(57,6)$ \\
\hline & Completo & $1436(80,9)$ \\
\hline & Incompleto & $340(19,1)$ \\
\hline \multicolumn{3}{|c|}{ Renda Salarial } \\
\hline & 1-3 salários mínimos & $883(49,7)$ \\
\hline & 3-5 salários mínimos & $554(31,2)$ \\
\hline & >5 salários mínimos & $339(19,1)$ \\
\hline
\end{tabular}

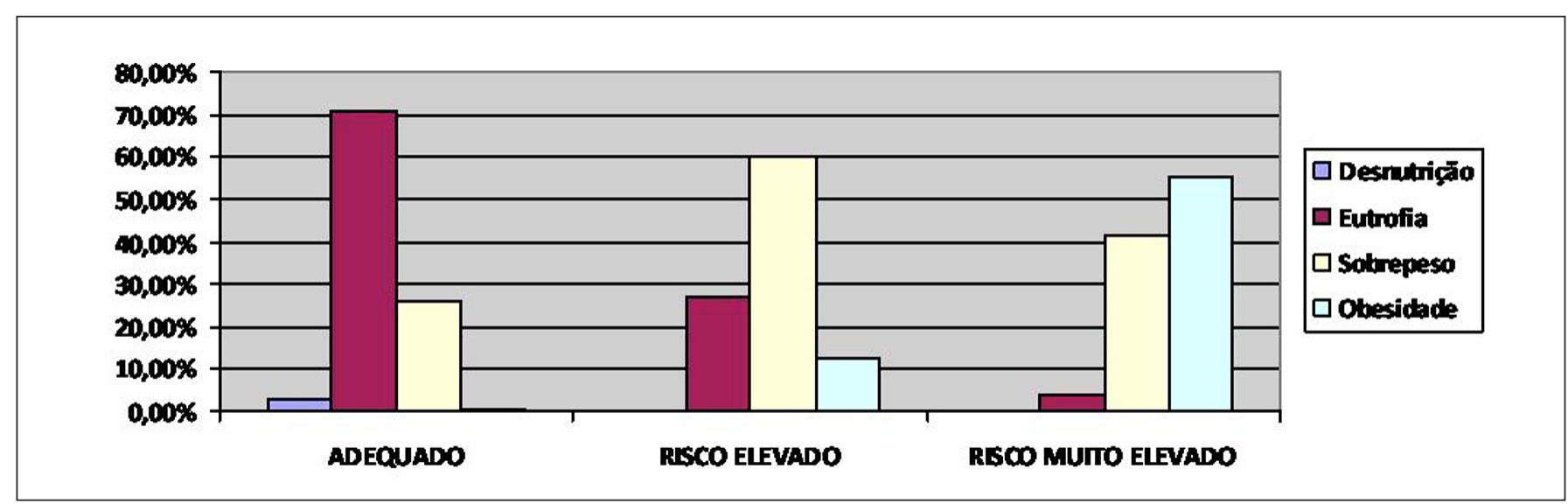

Figura 1 - Correlação entre classificação de IMC e classificação da circunferência da cintura.

Os indivíduos do estudo que relataram ter diabetes mellitus apresentaram relação significativa com a classificação do IMC e da circunferência da cintura $(p=0,011$ e $p<0,0001$, respectivamente), sendo que a maioria dos diabéticos encontrava-se em sobrepeso e obesidade $(44,7 \%$ e $42,1 \%$, respectivamente) pelo IMC e classificação de risco muito elevado para circunferência da cintura $(60,5 \%)$. Nos hipertensos, também foi observada diferença significativa para os 2 parâmetros de avaliação nutricional. A maioria dos hipertensos $(50,8 \%)$ era obesa $(p<0,0001)$ e $68,2 \%$ apresentavam risco muito elevado na classificação da circunferência da cintura $(p<0,0001)$. Da mesma forma, o sobrepeso e a obesidade foram mais prevalentes nos dislipidêmicos $(40,5 \%$ e $34,6 \%$, respectivamente) e nos indivíduos com classificação de risco muito elevado na circunferência da cintura (51\%), apresentando diferença significativa $(p=0,002$ e $p<0,0001)$. 
O hipotireoidismo também apresentou diferença significativa na correlação com a classificação da circunferência da cintura $(p<0,0001)$. Cinquenta e nove por cento dos indivíduos que apresentavam essa doença tinham a classificação de risco muito elevado. Com relação à depressão, o sobrepeso e obesidade também foram mais prevalentes, demonstrando diferença significativa $(p=0,02)$. Não observamos diferenças estatisticamente significativas para doenças cardiovasculares e câncer.

Quarenta e quatro (2,5\%) indivíduos tinham histórico de realização de cirurgia bariátrica e, nas comparações com as classificações avaliadas, notou-se diferença significativa apenas na classificação do risco pela circunferência da cintura ( $p=0,027)$, onde $40,9 \%$ dos pacientes que realizaram a cirurgia bariátrica tiveram classificação de risco elevado e $36,4 \%$ de risco muito elevado.

Com relação à prática de atividade física, 35,5\% da amostra se mostraram ativos fisicamente e, deste total, observamos diferença significativa entre as classificações do IMC e da circunferência da cintura $(p=0,022$ e $p<0,0001$, respectivamente), onde a maioria dos participantes da pesquisa que realizavam atividade física estava em sobrepeso, seguido de eutrofia (40,3\% e $39 \%$, respectivamente), enquanto $48,2 \%$ dos ativos fisicamente estavam com a circunferência da cintura na classificação adequada. Comparando essas classificações com o tempo de prática, foi observada diferença significativa com o IMC $(p=0,021)$ e com a classificação da circunferência da cintura $(p<0,0001)$.

O IMC e a circunferência da cintura apresentaram diferença significativa conforme o grau de instrução $(p<0,0001$ e $p<0,0001$, respectivamente). A maioria dos indivíduos com sobrepeso tinha grau de instrução de primeiro grau, seguido do ensino superior e segundo grau $(45,5 \%, 40,5 \%$ e $39,6 \%$, respectivamente). Mas quando observamos a prevalência de eutrofia entre estes grupos, notou-se que esta condição está presente em $38,3 \%$ daqueles que relataram ensino superior, enquanto $30,8 \%$ dos eutróficos relataram o segundo grav e apenas $12,1 \%$ destes informaram o primeiro grau como o grau de instrução.

Outra condição que se apresentou com diferença significativa entre as classificações do IMC $(p<0,0001)$ e circunferência da cintura $(p<0,0001)$ foi o estado civil. Com relação ao IMC, os solteiros apresentaram maior prevalência de eutrofia $(40,3 \%)$, seguida de sobrepeso (39\%). Já os casados e divorciados se encontraram mais em sobrepeso (42,8\% e 35\%, respectivamente), enquanto os viúvos tiveram maior prevalência de obesidade $(46,2 \%)$. Na classificação da circunferência da cintura, os resultados foram similares. No grupo dos casados, divorciados e viúvos, a prevalência foi de risco muito aumentado $(42,3 \%, 50 \%, 53,8 \%$, respectivamente), enquanto $45,7 \%$ dos solteiros tinham a circunferência da cintura em classificação adequada.

\section{DISCUSSÃO}

A prevalência de excesso de peso e de obesidade cresceu drasticamente nos últimos anos, sendo caracterizada pela transição nutricional, onde observamos diminuição da desnutrição concomitante a esse aumento de sobrepeso e obesidade. Em 34 anos (de 1974-1975 a 2008-2009), o excesso de peso aumentou em quase três vezes no sexo masculino (de 18,5\% para $50,1 \%$ ) e em quase duas vezes no sexo feminino (de $28,7 \%$ para $48 \%$ ), enquanto a obesidade aumentou em mais de quatro vezes para homens (de 2,8\% para 12,4\%) e em mais de duas vezes para mulheres (de $8 \%$ para $16,9 \%)^{7}$. No que tange à cidade de São Paulo, segundo o Boletim ISA Capital, com 4.043 entrevistados, a população adulta apresenta $36,2 \%$ de sobrepeso e $20,5 \%$ de obesidade ${ }^{17}$.

Estudos realizados em outras regiões do Brasil também observam estes resultados. No hospital universitário de Petrolina-PE, com 214 servidores avaliados, a frequência de sobrepeso e obesidade foi de $49,5 \%{ }^{18}$. Já em LondrinaPR, esse número aumentou para 64\%, na avaliação de 380 servidores do Hospital Universitário de Londrina ${ }^{19}$. No estudo de Simon et al. ${ }^{20}$, com colaboradores de uma unidade de alimentação e nutrição, os autores observaram média de ganho de peso de 500 gramas a cada ano trabalhado.

Embora o presente estudo não tenha avaliado a ingestão alimentar, sabe-se que a inadequação alimentar é um fator importante para o desenvolvimento do excesso de peso e obesidade. Mesmo observando aumento no consumo regular de frutas e hortaliças de 33\%, em 2008, para 35,2\%, em 2016, na população brasileira, este número ainda é pequeno, já que apenas 1 entre 3 adultos consomem frutas e hortaliças 5 dias da semana. Além disso, a POF de 20082009 demonstra que o consumo excessivo de açúcar ocorre em $61 \%$ da população, de gordura saturada (maior do que $7 \%$ do consumo de energia), em $82 \%$, e de sódio em mais de $70 \%$, em contrapartida, $68 \%$ consomem abaixo do recomendado de fibras ${ }^{7}$.

Quanto à circunferência da cintura, foram encontrado $25,1 \%$ da população com risco elevado e $37,2 \%$ com risco muito elevado para doenças cardiovasculares. No estudo de Maduro et al. ${ }^{18}$, a circunferência abdominal aumentada foi encontrada em $5 \%$ dos homens e $14 \%$ das mulheres, índice muito inferior em relação ao atual estudo, e também ao de Porto et al. ${ }^{19}$, onde $70,8 \%$ apresentaram risco moderado/ elevado para doenças cardiovasculares. Esta medida é utilizada como preditor de risco cardiovascular, uma vez que é capaz de refletir o acúmulo de gordura intra-abdominal ou visceral, que é considerada mais perigosa que a subcutânea, pelo potencial aterogênico ${ }^{21}$.

A obesidade abdominal também foi encontrada em indivíduos eutróficos, e esta possível combinação de excesso de adiposidade com massa magra diminuída é igualmente 
preocupante quanto ao IMC elevado, pois aponta para uma predisposição ao desenvolvimento de doenças similares a de obesos, como hipercolesterolemia, hipertensão, diabetes e doenças cardiovasculares ${ }^{18}$.

Quando correlacionamos o horário de trabalho com o estado nutricional, observamos que a maior prevalência de sobrepeso e obesidade esteve presente nos profissionais que trabalhavam no horário noturno, resultados similares aos encontrados por Maduro et al. ${ }^{18}$ e Simon et al. ${ }^{20}$. O trabalho neste horário apresenta importante ligação com fatores que podem potencializar o ganho de peso como maus hábitos alimentares e realização de refeições coletivas (utilizado como estratégia para inibir o sono e manter-se acordado durante o plantão). A restrição do sono e, consequentemente, alteração no ciclo circadiano pode contribuir para desregulação de secreções hormonais, ocasionando aumento do apetite, resistência à insulina e redução do gasto energético ${ }^{20}$.

Em 10 anos, houve aumento nacional em 61,8\% do diagnóstico de diabetes mellitus e de 14,2\% de hipertensão ${ }^{8}$. No estudo atual, foi possível reafirmar que diabetes mellitus, hipertensão arterial sistêmica, dislipidemia, depressão e hipotireoidismo estão associados com excesso de peso.

A cirurgia bariátrica é uma opção que pode auxiliar no tratamento da obesidade grave e as comorbidades que cercam esta doença. Contudo, ainda há poucos estudos a longo prazo com altas taxas de seguimento que possam concluir que, tanto a perda de peso como a melhoria da qualidade de vida são sustentadas. Cerca de $15 \%$ dos pacientes submetidos a cirurgia voltam a reganhar o peso. O reganho de peso é multifatorial e pode estar associado com deficiências nutricionais, ingestão de álcool, inatividade física, alterações hormonais e metabólicas, reaparecimento de comorbidades, problemas de saúde mental e abandono do acompanhamento multiprofissional, por exemplo. No atual estudo, podemos verificar que colaboradores que realizaram cirurgia bariátrica possuem maior circunferência da cintura em relação aos que não realizaram o procedimento. Entretanto, indivíduos que são submetidos a este tipo de tratamento, normalmente, apresentam graus mais elevados de obesidade. Vale ressaltar que a cirurgia bariátrica não finaliza o tratamento da obesidade, sendo necessário o acompanhamento por uma equipe multidisciplinar ${ }^{22}$.

Segundo a $\mathrm{OMS}^{23}$, adultos com idade entre 18 e 64 anos devem fazer pelo menos 150 minutos de atividade física aeróbia de intensidade moderada durante a semana. Para benefícios adicionais à saúde, os adultos devem aumentar sua atividade física aeróbica de intensidade moderada para 300 minutos por semana ${ }^{24}$. Na população estudada, apenas $35,5 \%$ realizavam atividade física e a maioria $(35,5 \%)$ fazia até 150 minutos de atividade, assim como os resultados encontrados por Maduro et al. ${ }^{18}$ e Porto et al. ${ }^{19}$.
A prevalência de excesso de peso aumenta continuamente em todos os estratos de renda para a população adulta masculina e, no caso da população feminina, o excesso de peso aumenta conforme menor a renda ${ }^{7}$. Também entre as mulheres, a frequência de excesso de peso diminui conforme o aumento do nível de escolaridade ${ }^{8}$. $O$ sobrepeso e a obesidade em classes de menor escolaridade e renda podem ser explicados pelo padrão de dieta, com alto conteúdo energético associado ao sedentarismo e à falta de informação.

Colaboradores casados, divorciados e viúvos estão associados com maior excesso de peso e circunferência da cintura aumentada em relação aos solteiros. Dankyau et al. ${ }^{25}$ observaram que indivíduos casados eram 4 vezes mais propensos a serem obesos do que os solteiros. $O$ estado civil pode impactar no peso e na circunferência da cintura devido às mudanças no comportamento social, que acarretam aumento de ingestão calórica, decorrente de alimentos ricos em gorduras e açúcares e diminuição de gasto energético, consequente da negligência às atividades físicas mais rigorosas e aumento da frequência à ida em restaurantes e lanchonetes como atividades de lazer. Não obstante, existe a tendência a diminuir a preocupação com a autoimagem ${ }^{25}$.

Programas de promoção da saúde no local de trabalho se mostraram vantajosos para a saúde física e mental dos colaboradores, com adoção de melhores hábitos de vida. Esses programas se mostraram benéficos também à empresa e à sociedade, pois têm foco na diminuição do risco de doenças crônicas não transmissíveis ${ }^{26}$.

\section{CONCLUSÃo}

Diante da alta prevalência encontrada de sobrepeso e obesidade, e risco para doenças cardiovasculares, na população estudada, faz-se necessária a criação de programas de educação nutricional com estratégias que promovam hábitos alimentares mais saudáveis e incentivo à prática de atividade física. Estas são ferramentas simples, mas que garantem mudanças no estilo de vida, garantindo a saúde física, prevenção de doenças crônicas, melhor qualidade de vida e desempenho profissional entre os trabalhadores.

Sugere-se, ainda, o desenvolvimento de novos estudos, incluindo análise de ingestão alimentar e métodos considerados padrão-ouro na avaliação da composição corporal, assim como aplicação de questionários de qualidade de vida.

\section{REFERÊNCIAS}

1. Zapata ME, Bibiloni MDM, Tur JA. Prevalence of overweight, obesity, abdominal-obesity and short stature of adult population of Rosario, Argentina. Nutr Hosp. 2016;33(5):580.

2. NCD Risk Factor Collaboration (NCD-RisC). Trends in adult body-mass index in 200 countries from 1975 to 2014: a pooled 
analysis of 1698 population-based measurement studies with 19.2 million participants. Lancet. 2016;387(10026):1377-96.

3. World Health Organization!. Global health risks: mortality and burden of disease attributable to selected major risks. Geneva: World Health Organization; 2009.

4. World Health Organization. Integrated chronic disease prevention and control. Geneva: World Health Organization; 2016.

5. Diez-Canseco F, Saavedra-Garcia L. Programas sociales y reducción de la obesidad en el Perú: reflexiones desde la investigación. Rev Peru Med Exp Salud Publica. 2017;34(1):105-12.

6. FAO, OMS, WFP, UNICEF. Panorama de la seguridad Alimentaria y Nutricional en América Latina y el Caribe. Santiago de Chile. 2018.

7. IBGE. Pesquisa de orçamentos familiares 2008-2009: análise do consumo alimentar pessoal no Brasil. Rio de Janeiro: IBGE; 2011.

8. Brasil. Vigitel Brasil 2018: vigilância de fatores de risco e proteção para doenças crônicas por inquérito telefônico: estimativas sobre frequência e distribuição sociodemográfica de fatores de risco e proteção para doenças crônicas nas capitais dos 26 estados brasileiros e no Distrito Federal em 2018. Brasília: Ministério da Saúde; 2018.

9. Volpe R, Stefano P, Massimiliano M, Francesca M, Gianluca S, Federica R. Healthy fats for healthy nutrition. An educational approach in the workplace to regulate food choices and improve prevention of non-communicable diseases. High Blood Press Cardiovasc Prev. 2015;22(4):395-401.

10. IBGE. Práticas de esporte e atividade física: 2015. Pesquisa Nacional por amostra domicílios. Rio de Janeiro: IBGE; 2017. $80 \mathrm{p}$.

11. BezerraIWL, OliveiraAG,PinheiroLGB, Morais CMM, Sampaio LMB. Evaluation of the nutritional status of workers of transformation industries adherent to the Brazilian Workers' Food Program. Acomparative study. PLoS One. 2017;12(2):e0171821.

12. Hunter RF, Gough A, Murray JM, Tang J, Brennan SF, Chrzanowski-Smith OJ, et al. Public Health Research. A loyalty scheme to encourage physical activity in office workers: a cluster RCT. Southampton: NIHR Journals Library; 2019.

13. Dias MCG, Horie LM, Catalani LA, Waitzberg DL. Exame físico e antropometria. In: Waitzberg DL, ed. Nutrição oral, enteral e parenteral na prática clínica. $5^{\mathrm{a}}$ ed. São Paulo: Atheneu; 2017. p.387-418.

14. World Health Organization. Obesity: preventing and managing the global epidemic. Geneva: World Health Organization; 2000.

15. Albala C, Lebrão ML, León Díaz EM, Ham-Chande R, Hennis AJ, Palloni A, et al. Encuesta Salud, Bienestar y Envejecimiento
(SABE): metodología de la encuesta y perfil de la población estudiada. Rev Panam Salud Pública. 2005;17(5/6):307-22.

16. World Health Organization. Physical activity. Geneva: World Health Organization; 2020. [cited 08 de junho de 2020]. Disponível em: https://www.who.int/es/news-room/fact-sheets/detail/ physical-activity

17. São Paulo (SP). Secretaria Municipal da Saúde. Coordenação de Epidemiologia e Informação - CEInfo. Boletim ISA Capital 2015, $\mathrm{n}^{\circ}$ 0, 2017: Aspectos metodológicos e produção de análises. São Paulo: CEInfo; 2017, 32p.

18. Maduro PA, Nascimento MM, Araújo IS, Barbosa LF, Pereira LGD. Análise do estado nutricional e nível de atividade física dos servidores do hospital universitário de Petrolina-PE. RBNE. 2017;11(67):856-67.

19. Porto DB, Arruda GA, Altimari LR, Cardoso Júnior CG. Autopercepção de saúde em trabalhadores de um Hospital Universitário e sua associação com indicadores de adiposidade, pressão arterial e prática de atividade física. Ciênc Saúde Coletiva. 2016;21(4):1113-22.

20. Simon MISS, Garcia CA, Lino ND, Forte GC, Fontoura ID, Oliveira ABA. Avaliação nutricional dos profissionais do serviço de nutrição e dietética de um hospital terciário de Porto Alegre. Cad Saúde Colet. 2014;22(1):69-74.

21. Huang JH, Huang SL, Li RH, Wang LH, Chen YL, Tang FC. Effects of nutrition and exercise health behaviors on predicted risk of cardiovascular disease among workers with different body mass index levels. Int J Environ Res Public Health. 2014;11(5):4664-75.

22. Busetto L, Dicker D, Azran C, Batterham RL, Farpour-Lambert N, Fried M, et al. Practical recommendations of the obesity management Task Force of the European Association for the Study of Obesity for the Post-Bariatric Surgery Medical Management. Obes Facts. 2017;10(6):597-632.

23. World Health Organization. Global recommendations on physical activity for health. Geneva: World Health Organization; 2011.

24. Prieske O, Dalager T, Herz M, Hortobagyi T, Sjogaard G, Sogaard $\mathrm{K}$, et al. Effects of physical exercise training in the workplace on physical fitness: a systematic review and meta-analysis. Sports Med. 2019;49(12):1903-21.

25. Dankyau M, Shu'aibu JA, Oyebanji AE, Mamven OV. Prevalence and correlates of obesity and overweight in healthcare workers at a tertiary hospital. J Med Tropics. 2016;18(2):55-9.

26. Kugathasan TA, Lecot F, Laberge S, Tremblay J, Mathieu M-E. Activate Your Health, a 3-year, multi-site, workplace healthy lifestyle promotion program: study design. BMC Public Health. 2019;19(1):1140.

Local de realização do estudo: A. C. Camargo Cancer Center, São Paulo, SP, Brasil.

Conflito de interesse: Os autores declaram não haver. 